\title{
Quiver Grassmannians associated with string modules
}

\author{
G. Cerulli Irelli
}

Received: 5 November 2009 / Accepted: 21 June 2010 / Published online: 14 July 2010

(C) Springer Science+Business Media, LLC 2010

\begin{abstract}
We provide a technique to compute the Euler-Poincaré characteristic of a class of projective varieties called quiver Grassmannians. This technique applies to quiver Grassmannians associated with "orientable string modules". As an application we explicitly compute the Euler-Poincaré characteristic of quiver Grassmannians associated with indecomposable pre-projective, pre-injective and regular homogeneous representations of an affine quiver of type $\tilde{A}_{p, 1}$. For $p=1$, this approach provides another proof of a result due to Caldero and Zelevinsky (in Mosc. Math. J. 6(3):411429, 2006).
\end{abstract}

Keywords Cluster algebras · Cluster character - Quiver Grassmannians · Euler characteristic $\cdot$ String modules

\section{Introduction and main results}

In this paper we provide a technique to compute the Euler-Poincaré characteristic of some complex projective varieties called quiver Grassmannians. In the last few years many authors have shown the importance of such projective varieties and of their Euler-Poincaré characteristic in the theory of cluster algebras (see $[5-7,16]$ ), introduced and studied by S. Fomin and A. Zelevinsky [18-20].

Given a quiver $Q$ and a $Q$-representation $M$, the quiver $\operatorname{Grassmannian} \operatorname{Gr}_{\mathbf{e}}(M)$ is the set of all sub-representations of $M$ of a fixed dimension vector e (see Sect. 1.1). This is a complex projective variety and our aim is to compute its Euler-Poincaré characteristic $\chi_{\mathbf{e}}(M)$. Our main result (Theorem 1) says that under some technical

Research supported by grant CPDA071244/07 of Padova University.

G. Cerulli Irelli $(\bowtie)$

Dipartimento di Matematica Pura ed Applicata, Università degli studi di Padova, Via Trieste 63,

35121 Padova, Italy

e-mail: giovanni.cerulliirelli@gmail.com 
hypotheses on $M$, there is an algebraic action of the one-dimensional torus $T=\mathbb{C}^{*}$ on $\mathrm{Gr}_{\mathbf{e}}(M)$. It is well-known (see Sect. 2) that if a complex projective variety is endowed with an algebraic action of a complex torus with finitely many fixed points, then its Euler-Poincaré characteristic equals the number of fixed points of this action and, in particular, it is positive. In general it is not true that the Euler-Poincaré characteristic of a quiver Grassmannian is positive (see [16, Example 3.6]) but it is proved in [23] for quiver Grassmannians associated with rigid representations of acyclic quivers, as conjectured in [18]. The fixed points of the action of $T$ on $\operatorname{Gr}_{\mathbf{e}}(M)$ are the "coordinate" subrepresentations of $M$ of dimension vector e (Sect. 1.2). As a combinatorial tool to count them, we consider the coefficient-quiver introduced by Ringel (see Sect. 1.3) and we notice that its successor closed subquivers are in bijection with coordinate subrepresentations of $M$ (Proposition 1).

We prove that "orientable string modules" (see Definition 1) satisfy the hypotheses of Theorem 1. Such a class of $Q$-representations includes (up to "right-equivalence") all the representations of the affine quiver of type $\tilde{A}_{p, 1}$ and most of the representations of the affine quiver of type $\tilde{A}_{p, q}$.

As an application we explicitly compute $\chi_{\mathbf{e}}(M)$ when $M$ is an indecomposable pre-projective, pre-injective and regular homogeneous representation of the affine quiver of type $\tilde{A}_{p, 1}$. We hence find another proof of results of [9] for $p=1$, and of [10] and [11] for $p=2$. Such computations can be used to have an explicit description of the bases of cluster algebras of type $\tilde{A}_{p, q}$ found in [11] and [17] and for further studies of such cluster algebras [12]. In addition it would be interesting to compare our computations with results of [22] where the authors compute the Laurent expansion of cluster variables of cluster algebras arising from surfaces. In particular this gives a technique to compute the Euler-Poincaré characteristic of quiver Grassmannians associated with rigid representations of quivers associated with triangulations of surfaces with marked points. This family includes quivers of type $\tilde{A}_{p, q}$ where our technique applies. In type $A$ one can compare our results with results of [1].

To conclude the introduction we remark that having a torus action on a smooth projective variety $X$ gives rise to a cellular decomposition of $X([4,13])$. It is known that if $M$ is a rigid $Q$-representation (i.e. without self-extensions) then $\operatorname{Gr}_{\mathbf{e}}(M)$ is smooth [8]. In particular if $M$ is a rigid $Q$-representation satisfying hypothesis of Theorem 1 then $\operatorname{Gr}_{\mathbf{e}}(M)$ has a cellular decomposition. This approach is used in [12].

The paper is organized as follows: in Sect. 1.1 we recall some basic facts about quivers and quiver Grassmannians; in Sect. 1.2 we state our main result; in Sect. 1.3 we introduce the coefficient-quiver of a $Q$-representation and we show how to use it as a combinatorial tool to apply the main result; in Sect. 1.4 we introduce orientable string modules and we prove that they satisfy the hypotheses of our main theorem; in Sect. 1.5 we give an explicit application for quivers of type $\tilde{A}_{p, 1}$. All the remaining sections are devoted to proofs.

\subsection{Quiver Grassmannians}

We recall the definition of quiver Grassmannians. Given a quiver $Q=\left(Q_{0}, Q_{1}\right)$, i.e. an oriented graph with vertex set $Q_{0}=\{1, \ldots, n\}$ and arrow set $Q_{1}$, a $Q$-representation $M$ consists of a collection of complex vector spaces $\left\{M(i), i \in Q_{0}\right\}$ and a collection of linear maps $\left\{M(a): M(j) \rightarrow M(i) \mid a: j \rightarrow i \in Q_{1}\right\}$. 
Table 1 Some $Q$-representations and their coefficient-quiver. In the fourth row, we denote by $J_{2}(0)$ the $2 \times 2$ nilpotent Jordan block. In the last two rows $E_{i j}$ denotes the $4 \times 4$ elementary matrix with 1 in the $i j$-component and zero elsewhere

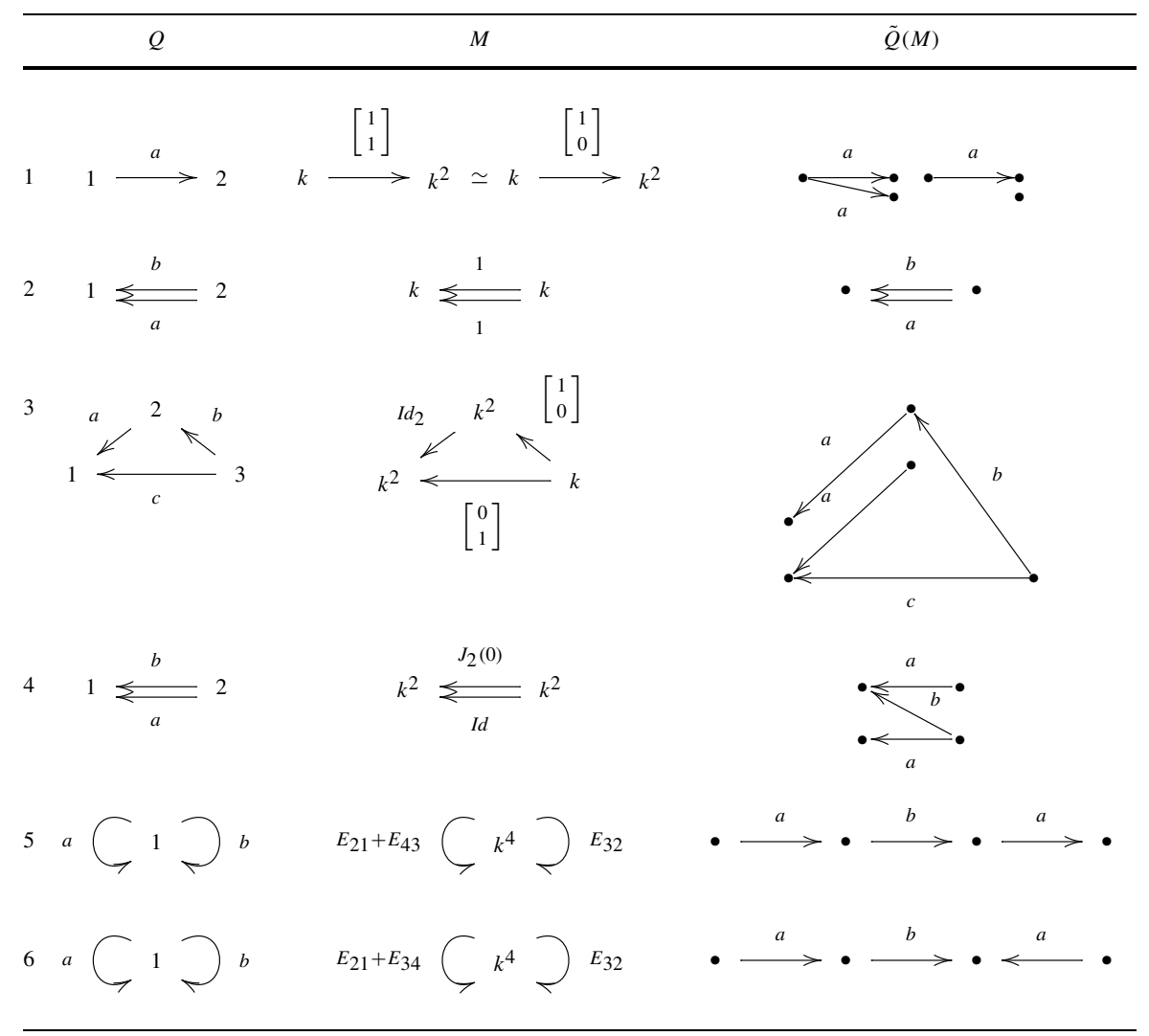

Example 1 The first column of Table 1 shows some examples of quivers $Q$ and the second one shows an example of a $Q$-representation $M$. We denote by $k$ the field of complex numbers. In the last two rows we use the notation $E_{i, j}$ to denote the linear operator on $k^{4}$ which sends the $j$ th basis vector to the $i$ th one and fixes all the others.

A subrepresentation $N$ of $M$ consists of a collection of vector subspaces $N(i)$ of $M(i), i \in Q_{0}$, such that $M(a) N(j) \subset N(i)$ for every arrow $a: j \rightarrow i$ of $Q$. For example the $Q$-representation $M$ shown in the first line of Table 1 does not admit the $Q$-representation $(k \longrightarrow 0)$ as its subrepresentation (because the map $M(a)$ has one-dimensional image) but admits $(0 \longrightarrow k)$.

The dimension vector of $M$ is the vector $\operatorname{dim}(M):=\left(\operatorname{dim}_{\mathbb{C}}(M(i)): i \in Q_{0}\right)$ where $\operatorname{dim}_{\mathbb{C}}(M(i))$ denotes the complex dimension of the vector space $M(i)$. For example in Table 1 the dimension vector of $M$ is respectively, from above to below, $(1,2)$, $(1,1),(2,2,1),(2,2),(4),(4)$.

The path algebra $k Q$ of $Q$ is the complex vector space with as basis the paths of $Q$ (i.e. concatenations of arrows) endowed with the multiplication given by the 
juxtaposition of paths. It is known (see e.g. [3]) that the category of $Q$-representations is equivalent to the category of $k Q$-modules. In particular every $Q$-representation can be seen as a $k Q$-module and viceversa every $k Q$-module has a natural structure of $Q$-representation.

Finally, the quiver Grassmannian $\operatorname{Gr}_{\mathbf{e}}(M)$ of $M$ of dimension $\mathbf{e}=\left(e_{i}: i \in Q_{0}\right)$ is defined as the set of all the subrepresentations of $M$ of dimension vector $\mathbf{e}$, that is,

$$
\operatorname{Gr}_{\mathbf{e}}(M):=\{N \subset M: \operatorname{dim}(N)=\mathbf{e}\}
$$

Example 2 For the $Q$-representations $M$ shown in lines 1 and 2 of Table 1 the quiver Grassmannian $\operatorname{Gr}_{(1,1)}(M)$ is a point. If $M$ is the $Q$-representation of line 3, $\operatorname{Gr}_{(1,1,1)}(M)$ is the empty set. Let $M$ be the $Q$-representation shown in line 4 . Here $J_{2}(0)=E_{12}=\left[\begin{array}{ll}0 & 1 \\ 0 & 0\end{array}\right]$ is the $2 \times 2$ nilpotent Jordan block which sends the second basis vector to the first one. We consider the set $\operatorname{Gr}_{(1,1)}(M)$ of subrepresentations of $M$ of dimension vector $(1,1)$. This consists of lines in $k^{2}$ spanned by non-zero vectors $v=(\lambda, \mu)^{t} \in k^{2}$ such that $v$ and $J_{2}(0) v$ are linearly dependent. In other words a line spanned by $v$ is in $\operatorname{Gr}_{(1,1)}(M)$ if and only if $\operatorname{det}\left[\begin{array}{ll}\lambda & \mu \\ \mu & 0\end{array}\right]=-\mu^{2}=0$. Then $\operatorname{Gr}_{(1,1)}(M)$ is a point which is actually not reduced, indeed the tangent space at this point has dimension one (see e.g. [12]).

If $M$ is the $Q$-representation shown in line 5 we consider $\operatorname{Gr}_{(1)}(M)$ which consists of the lines of $k^{4}$ invariant under the linear operators $E_{21}+E_{43}$ and $E_{32}$. It is easy to see that this set consists only of the line spanned by the fourth basis vector. Similarly if $M$ is the $Q$-representation shown in the last row of Table $1, \operatorname{Gr}_{(1)}(M)$ consists only of one point: the line spanned by the third basis vector.

We notice that the quiver Grassmannian $\operatorname{Gr}_{\mathbf{e}}(M)$ is closed inside the product $\prod_{i \in Q_{0}} \mathrm{Gr}_{e_{i}}(M(i))$, where $\mathrm{Gr}_{e_{i}}(M(i))$ denotes the usual Grassmannian of all vector subspaces of $M(i)$ of dimension $e_{i}$, which is a projective variety. As a consequence, $\operatorname{Gr}_{\mathbf{e}}(M)$ is a complex projective variety. We denote by $\chi_{\mathbf{e}}(M)$ its Euler-Poincaré characteristic. In the examples shown above $\chi_{\mathbf{e}}(M)$ is one if $\mathrm{Gr}_{\mathbf{e}}(M)$ is a (double) point and zero if it is the empty set.

\subsection{The main result}

The following theorem is our main result.

Theorem 1 Let $M$ be a $Q$-representation and for every $i \in Q_{0}$ let $B(i)$ be a linear basis of $M(i)$ such that for every arrow $a: j \rightarrow i$ of $Q$ and every element $b \in B(j)$ there exists an element $b^{\prime} \in B(i)$ and $c \in k$ (possibly zero) such that

$$
M(a) b=c b^{\prime}
$$

Suppose that each $v \in B(i)$ and all its multiples $c v, c \in k^{*}$, is assigned a degree $d(c v)=d(v) \in \mathbb{Z}$ so that: 
(D1) for all $i \in Q_{0}$ all vectors from $B(i)$ have different degrees;

(D2) for every arrow $a: j \rightarrow i$ of $Q$, whenever $b_{1} \neq b_{2}$ are elements of $B(j)$ such that $M(a) b_{1}$ and $M(a) b_{2}$ are non-zero we have:

$$
d\left(M(a) b_{1}\right)-d\left(M(a) b_{2}\right)=d\left(b_{1}\right)-d\left(b_{2}\right) .
$$

Then

$$
\chi_{\mathbf{e}}(M)=\mid\left\{N \in \operatorname{Gr}_{\mathbf{e}}(M): N(i) \text { is spanned by a part of } B(i)\right\} \mid
$$

in particular $\chi_{\mathbf{e}}(M)$ is positive.

The hypothesis (1) says that every column and every row of the matrix $M(a)$ contains at most one entry different from zero.

The hypothesis (D2) can be replaced by saying that every arrow $a$ of $Q$ has a degree $d(a) \in \mathbb{Z}$ so that $d\left(b^{\prime}\right)=d(b)+d(a)$ whenever $M(a) b=c b^{\prime}$, for some nonzero coefficient $c \in k$.

The thesis (3) says that we need to count the number of "coordinate" subrepresentations i.e. those $N \in \operatorname{Gr}_{\mathbf{e}}(M)$ whose vector space $N(i)$ is a coordinate subspace in the basis $B(i)$ (i.e. is spanned by elements of $B(i)$ ).

Example 3 Let $Q$ be the quiver with only one vertex and no arrows. A $Q$-representation is just a vector space $V$ and the quiver Grassmannians are usual Grassmannians of vector subspaces. Let $\left\{v_{1}, \ldots, v_{n}\right\}$ be a basis of $V$. We assign degree $d\left(v_{i}\right):=i$ and the hypotheses of Theorem 1 are satisfied. Then, by Theorem 1 , $\chi\left(\mathrm{Gr}_{k}(V)\right)$ is the number of coordinate vector subspaces (i.e. generated by basis vectors) of $V$ of dimension $k$. We hence find the well-known result: $\chi\left(\operatorname{Gr}_{k}(V)\right)=\left(\begin{array}{l}n \\ k\end{array}\right)$.

Let us give other examples with the help of Table 1 . The $Q$-representations shown in line 1 are isomorphic, but the first one does not satisfy the hypothesis (1) and we cannot apply Theorem 1 , while the second one does.

The second line shows an interesting example. The $Q$-representation $M$ of this line is a "deformation" of $M^{\prime}:=k \frac{1}{0} k$ and they have the same quiver Grassmannians (see Lemma 4). These two $Q$-representations are indeed right-equivalent in the sense of [15]. Theorem 1 applies to $M^{\prime}$ and we can hence compute $\chi_{\mathbf{e}}(M)$.

In line 3 of Table 1 we choose $d(a)=d(b):=0$ and $d(c):=1$ and hence the choice of a degree for the generator of the one-dimensional vector space at vertex 3 determines the choice of a degree for the two basis vectors at vertices 2 and 3 and these two degrees are different. We can hence apply Theorem 1.

In line 4 we choose $d(a):=0$ and $d(b):=1$.

In line 5 we choose $d(a)=d(b)=1$.

In line 6 we choose $d(a)=1$ and $d(b)=2$.

\subsection{Coefficient-quiver}

In order to compute $\chi_{\mathbf{e}}(M)$ with the help of Theorem 1 one can use a combinatorial tool called the coefficient-quiver $\tilde{Q}(M, B)$ of $M$ in the basis $B$ (introduced by Ringel 
in [24]). Let us recall its definition and show its utility. Let $M$ be a $Q$-representation and $B=\bigcup_{i \in Q_{0}} B(i)$ a collection of basis $B(i)$ of $M(i)$. The set $B$ is hence a basis of the vector space $\bigoplus_{i \in Q_{0}} M(i)$ and we refer to it as a basis of $M$. The coefficientquiver $\tilde{Q}(M, B)$ is a quiver whose vertices are identified with the elements of $B$; the arrows are defined as follows: for every arrow $a: j \rightarrow i$ of $Q$ and every element $b \in B(j)$ we expand $M(a) b=\sum c_{b^{\prime}} b^{\prime}$ in the basis $B(i)$ of $M(i)$ and we put an arrow (still denoted by $a$ ) from $b$ to $b^{\prime} \in B(i)$ in $\tilde{Q}(M, B)$ if the coefficient $c_{b^{\prime}}$ of $b^{\prime}$ in this expansion is non-zero. Table 1 shows examples of coefficient-quivers (which are denoted simply by $\tilde{Q}(M)$ since they are in the basis in which $M$ is presented).

We denote by $T \vec{\subset} \tilde{Q}(M)$ a successor closed subquiver $T$ of $\tilde{Q}(M)$, i.e. a subquiver $T$ such that if $j \in T_{0}$ is one of its vertices and $a: j \rightarrow i$ is an arrow of $\tilde{Q}(M)$ then $a$ is an arrow of $T$.

It is easy to see that the following proposition is equivalent to Theorem 1.

Proposition 1 Let $M$ be a $Q$-representation satisfying hypotheses of Theorem 1. Then

$$
\chi_{\mathbf{e}}(M)=\left|\left\{T \vec{\subset} \tilde{Q}(M):\left|T_{0} \cap B(i)\right|=e_{i}, \forall i \in Q_{0}\right\}\right|
$$

where $T_{0}$ denotes the vertices of $T$. In particular $\chi_{\mathbf{e}}(M)$ is positive.

For example let us consider the $Q$-representation $M$ shown in the third line of Table 1 . We have already noticed that $M$ satisfies hypotheses of Theorem 1 . Then we apply Proposition 1 and we find $\chi_{(1,0,0)}(M)=2$. Indeed there are two successor closed subquivers of $\tilde{Q}(M)$ with $\left|T_{0} \cap B(1)\right|=2$ and $\left|T_{0} \cap B(2)\right|=\left|T_{0} \cap B(3)\right|=0$ which are the two sinks (this is consistent with the fact that $\operatorname{Gr}_{(1,0,0)}(M)=\mathbb{P}^{1}\left(k^{2}\right)$ is a projective line). Many other examples can be taken from Table 1.

\subsection{String-modules}

We now show a class of $Q$-representations which satisfy the hypotheses of Theorem 1 .

A $Q$-representation $M$ is called a string module if it admits a basis $B_{0}$ such that the coefficient-quiver $\tilde{Q}\left(M, B_{0}\right)$ in this basis is a chain (i.e. a 2-regular graph not necessarily connected) and if every column and every row of every matrix $M(a)$ in this basis $B_{0}$ has at most one non-zero entry, i.e. it satisfies (1). We remark that this definition follows [14] but not [24] where (1) is not required. For a string module $M$ we sometimes avoid mentioning the basis $B_{0}$ and we denote the corresponding coefficient-quiver simply by $\tilde{Q}(M)$. The $Q$-representations shown in Table 1 are all string modules except the second one. It can be shown that a string module $M$ is indecomposable if and only if $\tilde{Q}(M)$ is connected ([14], [21, Sects. 3.5 and 4.1]).

Given an indecomposable string module $M$, the chain $\tilde{Q}(M)$ has two extreme vertices (i.e. joined with exactly one vertex). We say that two arrows of $\tilde{Q}(M)$ have the same orientation if they both point toward the same extreme vertex and they have different orientation otherwise. For example the two arrows labeled by $a$ in lines 5 and 6 of Table 1 have the same orientation in the line 5 while they have different orientation in the line 6 . 
During private conversations with J. Schröer we were introduced to the following definition.

Definition 1 A string module $M$ is called orientable if for every arrow $a$ of $Q$, all the corresponding arrows $a$ of $\tilde{Q}(M)$ have the same orientation.

For example line 5 of Table 1 shows an orientable string module while the line 6 shows a non-orientable one.

Proposition 2 If $M$ is an orientable string module then (4) holds.

In Sect. 2 we show that an orientable string module satisfies (1), (D1) and (D2) and hence, by Proposition 1, they satisfy (4).

1.5 Explicit computations in type $\tilde{A}_{p, 1}$

In this section we compute explicitly $\chi_{\mathbf{e}}(M)$ for some indecomposable representation $M$ of the affine quiver $Q_{p, 1}$ of type $\tilde{A}_{p, 1}$. Let us recall the definition of $Q_{p, 1}$.

Let $p \geq 1$ be an integer. By definition $Q_{p, 1}$ has one sink, one source and $p+1$ arrows which form two paths, one with $p$ arrows and the other with one arrow. We denote the vertices of $Q_{p, 1}$ by numbers from 1 to $p+1$ so that 1 is the sink, $p+1$ is the source and $k$ is joined to $k+1$ by the arrow $\varepsilon_{k}$, for $k=1,2, \ldots, p$ and $p+1$ is joined to 1 by the arrow $\varepsilon_{0}$ as shown below:

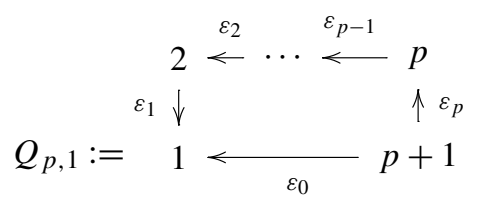

For every $n \geq 0$ and $1 \leq t \leq p$ we define the $Q_{p, 1}$-representations

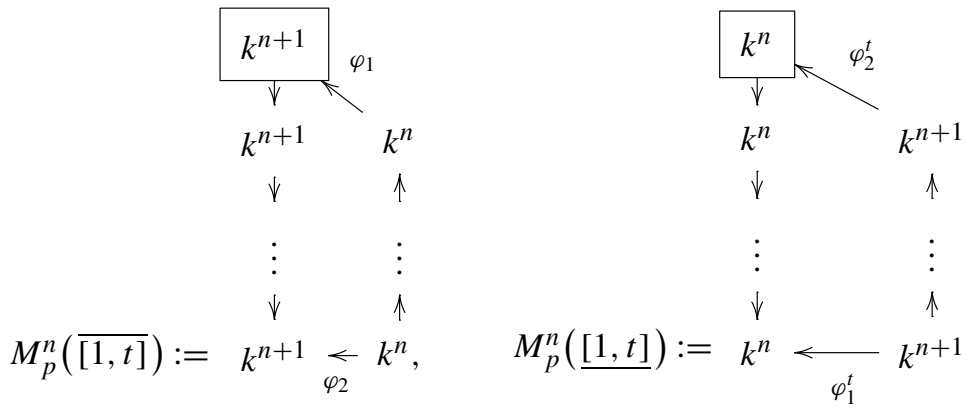

where the highlighted vector spaces correspond to the vertex $t$. These representations are called respectively pre-projective and pre-injective modules (see e.g. [2]). 
For every $\lambda \in k$ and $n \geq 1$, let $\operatorname{Reg}_{p}^{n}(\lambda)$ be the $Q_{p, 1}$-representation

$$
\begin{aligned}
& \begin{aligned}
& = \\
k^{n} & <
\end{aligned} \\
& =\downarrow \quad \uparrow= \\
& \operatorname{Reg}_{p}^{n}(\lambda):=k^{n} \leftarrow \frac{}{J_{n}(\lambda)} k^{n}
\end{aligned}
$$

with a Jordan block $J_{n}(\lambda)$ of eigenvalue $\lambda$ at the arrow $\varepsilon_{0}$ and the identity map in all the other arrows. This representation is called regular homogeneous. It is easy to see that $M_{p}^{n}(\overline{[1, t]}), M_{p}^{n}(\underline{[1, t])})$ and $\operatorname{Reg}_{p}^{n}(0)$ are orientable string modules (see Lemma 2) and $\chi_{\mathbf{e}}\left(\operatorname{Reg}_{p}^{n}(\lambda)\right)=\chi_{\mathbf{e}}\left(\operatorname{Reg}_{p}^{n}(0)\right)$ for every $\lambda \in k$ (Sect. 4.2). We can hence apply Theorem 1 (or Proposition 2).

We often use the following notation:

$$
\chi_{\mathbf{e}}([r, s]):=\prod_{k=r}^{s-2}\left(\begin{array}{c}
e_{k}-e_{s} \\
e_{k+1}-e_{s}
\end{array}\right)=\prod_{k=r+1}^{s-1}\left(\begin{array}{c}
e_{r}-e_{k+1} \\
e_{k}-e_{k+1}
\end{array}\right)
$$

with the convention that this product equals one whenever $r>s-2$. We interpret $\chi_{\mathbf{e}}([r, s])$ as the Euler characteristic of the flag variety

$$
\left\{k^{e_{r}} \supseteq M_{r+1} \supseteq \cdots \supseteq M_{s-1} \supseteq k^{e_{s}} \mid \operatorname{dim}\left(M_{k}\right)=e_{k}\right\} .
$$

Proposition 3 For every $n \geq 1,1 \leq t \leq p$ and $\lambda \in k$ we have

$$
\begin{aligned}
& \chi_{\left(e_{1}, \ldots, e_{p+1}\right)}\left(M_{p}^{n}(\overline{[1, t]})\right) \\
& =\left(\begin{array}{c}
e_{1}-1 \\
e_{p+1}
\end{array}\right)\left(\begin{array}{c}
n+1-e_{t} \\
e_{1}-e_{t}
\end{array}\right)\left(\begin{array}{c}
n+1-e_{t+1} \\
e_{t}-e_{t+1}
\end{array}\right)\left(\begin{array}{c}
n-e_{p+1} \\
e_{t+1}-e_{p+1}
\end{array}\right) \\
& \quad \times \chi_{\mathbf{e}}([1, t]) \chi_{\mathbf{e}}([t+1, p+1]) \\
& \chi_{\left(e_{1}, \ldots, e_{p+1}\right)}\left(M_{p}^{n}(\underline{[1, t]})\right) \\
& =\left(\begin{array}{c}
n-e_{p+1} \\
e_{1}-e_{p+1}
\end{array}\right)\left(\begin{array}{c}
e_{t+1} \\
e_{p+1}
\end{array}\right)\left(\begin{array}{c}
e_{t}+1 \\
e_{t+1}
\end{array}\right)\left(\begin{array}{c}
e_{1} \\
e_{t}
\end{array}\right) \chi_{\mathbf{e}}([1, t]) \chi_{\mathbf{e}}([t+1, p+1]), \\
& \chi_{\mathbf{e}}\left(\operatorname{Reg}_{p}^{n}(\lambda)\right)=\left(\begin{array}{c}
e_{1} \\
e_{p+1}
\end{array}\right)\left(\begin{array}{c}
n-e_{p+1} \\
e_{1}-e_{p+1}
\end{array}\right) \chi_{\mathbf{e}}([1, p+1]) .
\end{aligned}
$$

We always use the convention that the binomial coefficient $\left(\begin{array}{l}p \\ q\end{array}\right)$ equals 0 if $q<0$, $p<0, q>p$ and it equals 1 if $q=0$ and $p \geq q$.

\section{Proof of Theorem 1}

The proof is based on the following well-known fact: given a complex projective variety $X$ and an algebraic action $\varphi: T \times X \rightarrow X,(\lambda, x) \mapsto \lambda . x$ of the onedimensional torus $T=\mathbb{C}^{*}$ with finitely many fixed points, then the number of fixed 
points equals the Euler-Poincaré characteristic $\chi(X)$ of $X$. To see this we consider the decomposition $X=X^{T} \bigsqcup Y$ of $X$ into the disjoint union of the set $X^{T}$ of fixed points of $\varphi$ and of their complement $Y:=X \backslash X^{T}$. Such sets are locally closed and hence $\chi(X)=\chi\left(X^{T}\right)+\chi(Y)$. The restriction of $\varphi$ to $Y$ defines a surjective morphism $\varphi: T \times Y \rightarrow Y$ whose fibers are all isomorphic to $\mathbb{C}^{*}$. It follows that $\chi(Y)=\chi\left(\mathbb{C}^{*}\right)=0$ and hence $\chi(X)=\chi\left(X^{T}\right)$ which equals the number of fixed points of $\varphi$.

We hence find a torus action on our quiver Grassmannians.

Let $M$ be a representation satisfying hypotheses (D1) and (D2) of the theorem. The torus $k^{*}$ acts on $M$ as follows:

$$
\lambda . b:=\lambda^{d(b)} b, \quad \lambda \in k^{*}
$$

for every element $b \in B$ of the basis $B$ extended by linearity to all the elements of $M$. This action extends to quiver Grassmannians:

Lemma 1 Let $U \in \operatorname{Gr}_{\mathbf{e}}(M)$ be a subrepresentation of $M$ of dimension vector $\mathbf{e}$. Then, given $\lambda \in \mathrm{k}^{*}$, the set $\lambda . U:=\{\lambda . u \mid u \in U\}$ is a subrepresentation of $M$ of the same dimension vector $\mathbf{e}$ of $U$.

Proof Given an arrow $a: j \rightarrow i$ of $Q$ we define the number $d(a):=d(M(a) b)-$ $d(b)$ for an element $b \in B(j)$ such that $M(a) b$ is non-zero. This definition is independent of the choice of $b$ in view of (D2). Then it is easy to verify that for every $v \in M(j)$

$$
\lambda .(M(a) v)=\lambda^{d(a)} M(a)(\lambda . v)
$$

which concludes the proof.

Given a subrepresentation $U \in \operatorname{Gr}_{\mathbf{e}}(M)$, the element $\lambda \in k^{*}$ acts on each vector subspace $U(i)$ as a diagonal operator with different eigenvalues, in view of property (D1). Then the fixed subrepresentations $U=\lambda . U \in \mathrm{Gr}_{\mathbf{e}}(M)$ are precisely the coordinate subspaces of $M$ in the basis $B$ of dimension $e:=\sum_{i} e_{i}$ which concludes the proof of Theorem 1 .

\section{Proof of Proposition 2}

We prove that an orientable string module $M$ satisfies the hypotheses of Theorem 1 . By definition there exists a basis $B_{0}$ of $M$ so that (1) is satisfied and the coefficientquiver $\tilde{Q}\left(M, B_{0}\right)$ in $B_{0}$ is a chain. We have to assign a degree $d(b) \in \mathbb{Z}$ to the elements of $B_{0}$ (which are also the vertices of $\tilde{Q}\left(M, B_{0}\right)$ ) so that (D1) and (D2) are satisfied.

Since $S:=\tilde{Q}\left(M, B_{0}\right)$ is a chain we number the vertices of $S$ as $s_{1}, s_{2}, \ldots$ in such a way that for every $i=1, \ldots, m$ there is a unique edge $\varepsilon_{i}$ between $s_{i}$ and $s_{i+1}$. We assign the degree $d\left(s_{i}\right):=i$ for $i=1,2, \ldots$ Then (D1) is clearly satisfied (all the elements of $B_{0}$ have different degrees and hence all the elements of $B_{0}(i)$ have different degrees). Since $M$ is orientable it is also easy to prove that (D2) is satisfied. 
Indeed, by definition, for every arrow $a$ of $Q$ all the corresponding arrows $a$ of $S$ have all the same orientation, either all of them are oriented from $s_{i}$ to $s_{i+1}$ or from $s_{i+1}$ to $s_{i}$.

\section{Proof of Proposition 3}

For the convenience of the reader we prove Proposition 3 first in the case $p=1$ (the Kronecker quiver) and hence for $p \geq 1$.

All the proofs are based on the following lemma.

Lemma $2 M_{p}^{n}(\overline{[1, t]}), M_{p}^{n}(\underline{[1, t]})$ and $\operatorname{Reg}_{p}^{n}(0)$ are orientable string modules (in the sense of Definition 1). In particular (4) holds.

Proof All the linear maps defining such $Q_{p, 1}$-representations satisfy (1). It remains to show that their coefficient-quiver is a chain.

Let $S_{\varepsilon_{0}}$ be the subquiver of $Q_{p, 1}$ obtained by removing the arrow $\varepsilon_{0}$. We join together $n$ copies of $S_{\varepsilon_{0}}$ by using the arrow $\varepsilon_{0}$ and we get a string that we denote by $S_{0}^{n}$. The coefficient-quiver of $\operatorname{Reg}_{p}^{n}(0)$ is $S_{0}^{n}$ which is a chain.

Let $1 \leq t \leq p$ be a vertex of $Q_{p, 1}$. We consider the full subquiver $S(\overline{[1, t]})$ of $Q_{p, 1}$ with vertex set all the vertices $1,2, \ldots, t$. We join the string $S_{0}^{n}$ with the string $S([1, t])$ by using the arrow $\varepsilon_{0}$ and we get a new string that we call $S^{n}(\overline{[1, t]})$. Such a string is the coefficient-quiver of $M_{p}^{n}(\overline{[1, t]})$

In order to get the coefficient-quiver of $M_{p}^{n}([1, t])$ we proceed similarly: we consider the full subquiver $S([1, t])$ with vertices $t+1, t+2, \ldots, p, p+1$. We join $S([1, t])$ with $S^{n}$ by using the arrow $\varepsilon_{0}$ and we get a quiver $S^{n}([1, t])$. Such a quiver is the coefficient-quiver of $M_{p}^{n}([1, t])$. Figure 1 shows the case $p=4$, $t=n=3$.

\subsection{Type $\tilde{A}_{1,1}$ : the Kronecker quiver}

In this section we consider the Kronecker quiver $Q_{1,1}:=1 \stackrel{\varepsilon_{1}}{\longleftarrow} 2$ and its representations over the field $k$ of complex numbers. Let $\varphi_{1}, \varphi_{2}: k^{n} \rightarrow k^{n+1}$ be respectively the immersion in the vector subspace spanned by the first and by the last $n$ basis vectors. For every $n \geq 0$ and $\lambda \in k$ we consider the representations

$$
\begin{gathered}
M_{1}^{n}(\overline{[1,1]}):=k^{n+1} \underset{\varphi_{2}}{\longleftarrow} k^{n} ; \quad M_{1}^{n} \underline{\varphi_{1}[1,1]}:=k^{n} \underset{\varphi_{2}^{t}}{\longleftarrow} k^{n+1} ; \\
\operatorname{Reg}_{1}^{n}(\lambda):=k^{n} \underset{J_{n}(\lambda)}{\longleftarrow} k^{n} .
\end{gathered}
$$

The next result is contained in [9]. We give a slightly different proof by using Theorem 1 . 

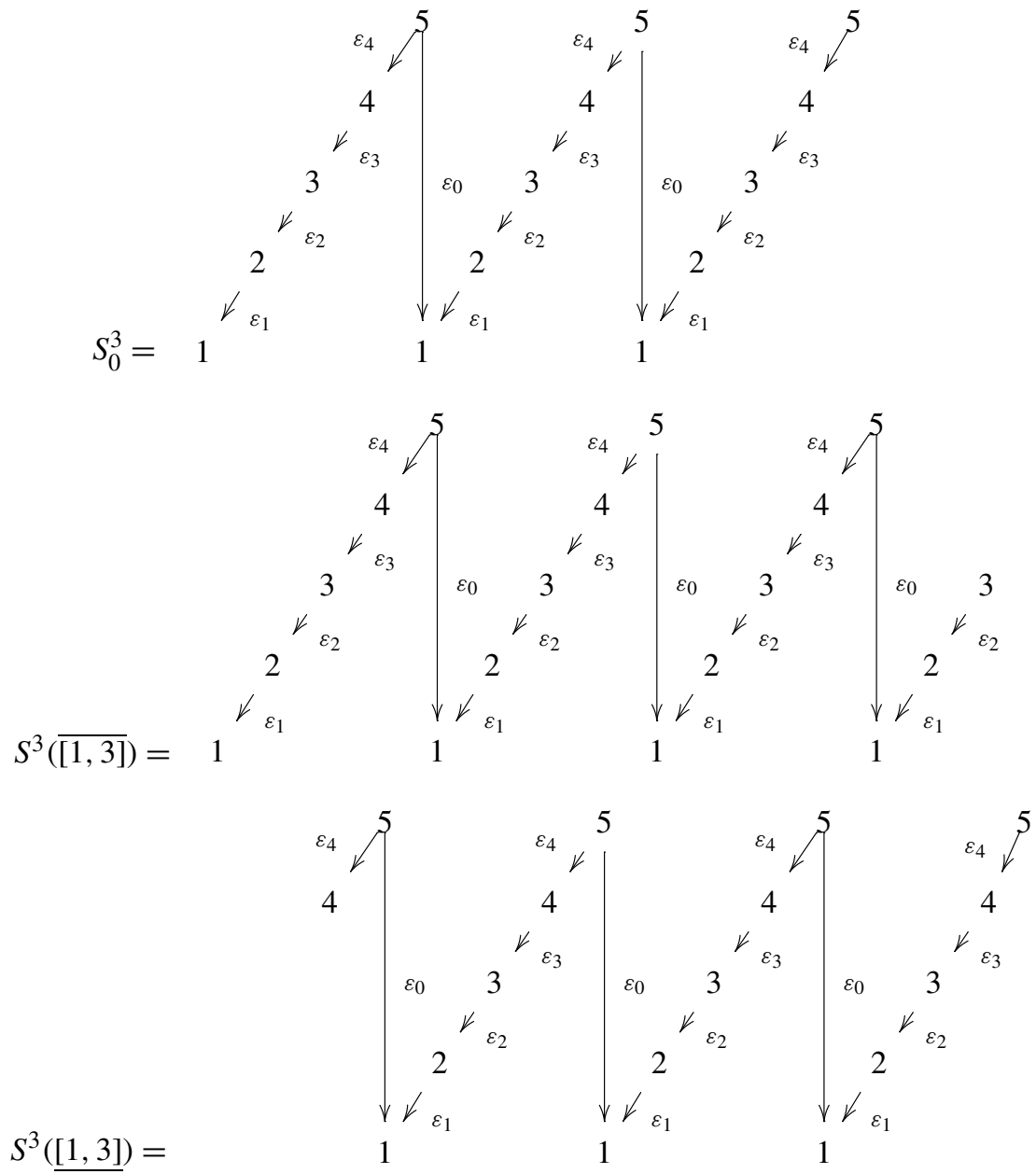

Fig. 1 The coefficient-quiver of $\operatorname{Reg}_{4}^{3}(0), M_{4}^{3}(\overline{[1,3]})$ and $M_{4}^{3}(\underline{[1,3]})$ respectively

Proposition 4 [9, Propositions 4.3 and 5.3] For every dimension vector $\mathbf{e}=\left(e_{1}, e_{2}\right)$ and $n \geq 0$ we have:

$$
\begin{aligned}
& \chi_{\left(e_{1}, e_{2}\right)}\left(M_{1}^{n}(\overline{[1,1]})\right)=\left(\begin{array}{c}
n+1-e_{2} \\
n+1-e_{1}
\end{array}\right)\left(\begin{array}{c}
e_{1}-1 \\
e_{2}
\end{array}\right)+\delta_{e_{1}, 0} \delta_{e_{2}, 0}, \\
& \chi_{\left(e_{1}, e_{2}\right)}\left(M_{1}^{n}(\underline{[1,1]})\right)=\left(\begin{array}{c}
e_{1}+1 \\
e_{2}
\end{array}\right)\left(\begin{array}{c}
n-e_{2} \\
n-e_{1}
\end{array}\right)+\delta_{e_{1}, n} \delta_{e_{2}, n+1}
\end{aligned}
$$

where $\delta_{a, b}$ denotes the Kronecker delta. For every $\lambda \in k$ :

$$
\chi_{\left(e_{1}, e_{2}\right)}\left(\operatorname{Reg}_{1}^{n}(\lambda)\right)=\left(\begin{array}{l}
n-e_{2} \\
n-e_{1}
\end{array}\right)\left(\begin{array}{l}
e_{1} \\
e_{2}
\end{array}\right) .
$$


Proof We notice that (11) follows from (10). Indeed $M_{1}^{n}[1,1] \simeq D M_{1}^{n}(\overline{[1,1]})$ where $D=\operatorname{Hom}_{k}(\cdot, k)$ is the duality functor and the isomorphism follows by exchanging the two vertices. Then we have (see also [8, Sect. 1.2]):

$$
\chi_{\left(e_{1}, e_{2}\right)}\left(M_{1}^{n}(\underline{[1,1]})\right)=\chi_{\left(n+1-e_{2}, n-e_{1}\right)}\left(M_{1}^{n}(\overline{[1,1]})\right) .
$$

We hence prove (10). By Lemma 2, the representation $M_{1}^{n}(\overline{[1,1]})$ is an orientable string module and we can apply Theorem 1 . In order to compute $\chi_{\left(e_{1}, e_{2}\right)}\left(M_{1}^{n}(\overline{[1,1]})\right)$, we have hence to count couples $\left\{T_{1}, T_{2}\right\}$ of subsets $T_{1} \subset[1, n+1], T_{2} \subset[1, n]$ such that $\left|T_{i}\right|=e_{i}(i=1,2)$ and $\varphi_{1}\left(T_{2}\right) \subset T_{1}, \varphi_{2}\left(T_{2}\right) \subset T_{1}$ where $\varphi_{1}, \varphi_{2}:[1, n] \rightarrow$ $[1, n+1]$ are the two maps defined by $\varphi_{1}(k)=k$ and $\varphi_{2}(k)=k+1$ for $k=1,2, \ldots, n$ (here and in the sequel we use the notation $[1, m]:=\{1,2, \ldots, m\}$ ). We need the following lemma.

Lemma 3 [9, Proof of Proposition 4.3] Let $n$ and $r$ be positive integers such that $1 \leq r \leq n$. For an $r$-element subset $J$ of $[1, n]$ we denote by $c(J)$ the number of connected components of $J$ (i.e. the number of maximal connected intervals in $J$ ). The number of $r$-element subsets $J$ of $[1, n]$ such that $c(J)=c$ is $\left(\begin{array}{l}r-1 \\ c-1\end{array}\right)\left(\begin{array}{c}n+1-r \\ c\end{array}\right)$.

Proof A proof of Lemma 3 can be found in [9, Proof of Proposition 4.3].

We hence continue the proof of (10). The choice of an element $k \in[1, n]$ determines the choice of the two different elements $\varphi_{1}(k)$ and $\varphi_{2}(k)$ of $[1, n+1]$; in general the choice of a subset $T_{2}$ of $[1, n]$ of cardinality $e_{2}$ with $c$ connected components determines the choice of $c+e_{2}$ elements of [1,n+1]. Given such a set $T_{2}$, there are hence $\left(\begin{array}{c}n+1-\left(c+e_{2}\right) \\ e_{1}-\left(c+e_{2}\right)\end{array}\right)$ choices for the sets $T_{1}$ such that $\left\{T_{1}, T_{2}\right\}$ is a desired couple. If $e_{1}=e_{2}=0$ then $\chi_{(0,0)}\left(M_{1}^{n}(\overline{[1,1]})\right)=1$. We assume $e_{1} \geq e_{2} \geq 1$. By Lemma 3 the number of $e_{2}$-element subsets $T_{2}$ of $[1, n]$ with $c\left(T_{2}\right)=c$ equals $\left(\begin{array}{c}e_{2}-1 \\ c-1\end{array}\right)\left(\begin{array}{c}n+1-e_{2} \\ c\end{array}\right)$. The number of desired couples $\left\{T_{1}, T_{2}\right\}$ is hence

$$
\begin{aligned}
\chi_{\left(e_{1}, e_{2}\right)}\left(M_{1}^{n}(\overline{[1,1]})\right) & =\sum_{c=1}^{e_{1}-e_{2}}\left(\begin{array}{c}
n+1-\left(c+e_{2}\right) \\
e_{1}-\left(c+e_{2}\right)
\end{array}\right)\left(\begin{array}{c}
e_{2}-1 \\
c-1
\end{array}\right)\left(\begin{array}{c}
n+1-e_{2} \\
c
\end{array}\right) \\
& =\sum_{c=1}^{e_{1}-e_{2}}\left(\begin{array}{c}
e_{1}-e_{2} \\
c
\end{array}\right)\left(\begin{array}{c}
e_{2}-1 \\
c-1
\end{array}\right)\left(\begin{array}{c}
n+1-e_{2} \\
e_{1}-e_{2}
\end{array}\right) \\
& =\left(\begin{array}{c}
n+1-e_{2} \\
e_{1}-e_{2}
\end{array}\right) \sum_{c=1}^{e_{1}-e_{2}}\left(\begin{array}{c}
e_{1}-e_{2} \\
c
\end{array}\right)\left(\begin{array}{c}
e_{2}-1 \\
e_{2}-c
\end{array}\right) \\
& =\left(\begin{array}{c}
n+1-e_{2} \\
e_{1}-e_{2}
\end{array}\right)\left(\begin{array}{c}
e_{1}-1 \\
e_{2}
\end{array}\right) .
\end{aligned}
$$

In the second equality we have used the identity: $\left(\begin{array}{c}n+1-r-q \\ p-q\end{array}\right)\left(\begin{array}{c}n+1-r \\ q\end{array}\right)=\left(\begin{array}{c}p \\ q\end{array}\right)\left(\begin{array}{c}n+1-r \\ p\end{array}\right)$ with $q=c, p=e_{1}-e_{2}$ and $r=e_{2}$; in the last equality we have used the Vandermonde's identity: $\sum_{k}\left(\begin{array}{l}a \\ k\end{array}\right)\left(\begin{array}{c}b \\ c-k\end{array}\right)=\left(\begin{array}{c}a+b \\ c\end{array}\right)$. 


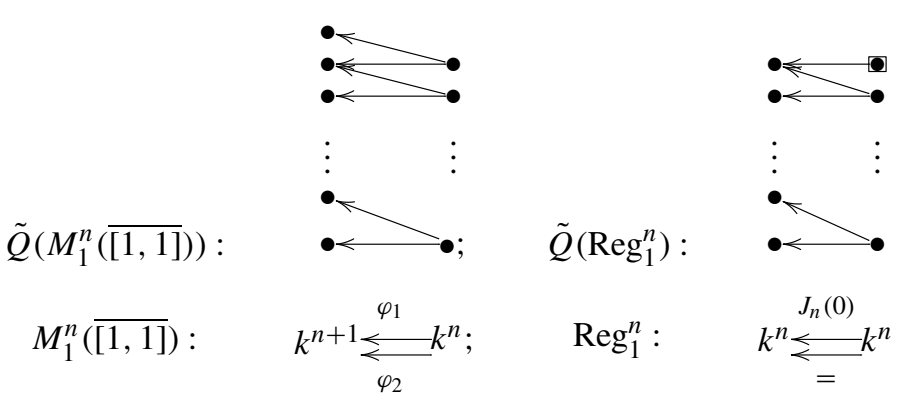

Fig. 2 Coefficient-quiver of $Q_{1,1}$-representations

We now prove (12). We first assume that $\lambda=0$. The representation $\operatorname{Reg}_{1,1}^{n}(0)$ is an orientable string module and we apply Theorem 1 . We prove (12) by induction on $n \geq 0$. For $n=0$ it is clear. Let hence $n \geq 1$. We have hence to count the number of couples $\left\{T_{1}, T_{2}\right\}$ of subsets $T_{2} \subset T_{1} \subset[1, n]$ such that $\left|T_{i}\right|=e_{i}$ and $J_{n}(0) T_{2} \subset T_{1} \cup 0$ where $J_{n}(0):[1, n] \rightarrow[1, n] \cup\{0\}$ maps $k$ to $k-1$ for $k=1,2, \ldots, n$. Alternatively, by Proposition 1, we can consider the coefficient-quiver $\tilde{Q}\left(\operatorname{Reg}_{1}^{n}\right)$ of $\operatorname{Reg}_{1}^{n}$ (shown in Fig. 2) and count its successor closed subquivers with $e_{1}$ sources and $e_{2}$ sinks. Such a subquiver either contains the unique vertex of $\tilde{Q}\left(\operatorname{Reg}_{1}^{n}\right)$ which is the source of a unique arrow (highlighted in Fig. 2) or it does not. Alternatively either $T_{2}$ contains $1=\operatorname{Ker}\left(J_{n}(0)\right)$ or it does not. We hence have

$$
\begin{aligned}
\chi_{\left(e_{1}, e_{2}\right)}\left(\operatorname{Reg}_{1}^{n}(0)\right) & =\chi_{\left(e_{1}-1, e_{2}-1\right)}\left(\operatorname{Reg}_{1}^{n-1}(0)\right)+\chi_{\left(e_{1}, e_{2}\right)}\left(M_{1}^{n-1}(\overline{[1,1]})\right) \\
& =\left(\begin{array}{l}
n-e_{2} \\
n-e_{1}
\end{array}\right)\left(\begin{array}{l}
e_{1}-1 \\
e_{2}-1
\end{array}\right)+\left(\begin{array}{c}
n-e_{2} \\
n-e_{1}
\end{array}\right)\left(\begin{array}{c}
e_{1}-1 \\
e_{2}
\end{array}\right)+\delta_{e_{1}, 0} \delta_{e_{2}, 0} \\
& =\left(\begin{array}{l}
n-e_{2} \\
n-e_{1}
\end{array}\right)\left(\begin{array}{l}
e_{1} \\
e_{2}
\end{array}\right)
\end{aligned}
$$

and we are done (we use the obvious fact that $\left.\left(\begin{array}{c}a-1 \\ b-1\end{array}\right)+\left(\begin{array}{c}a-1 \\ b\end{array}\right)=\left(\begin{array}{l}a \\ b\end{array}\right)-\delta_{a, 0} \delta_{b, 0}\right)$.

It remains to be considered the case where $\lambda \neq 0$ which is solved in the following lemma.

Lemma 4 For every $\lambda \in \mathbb{C}$ and $n \geq 1$ we have

$$
\chi_{\mathbf{e}}\left(\operatorname{Reg}_{1}^{n}(\lambda)\right)=\chi_{\mathbf{e}}\left(\operatorname{Reg}_{1}^{n}(0)\right)
$$

Proof As vector spaces, $\operatorname{Reg}_{1}^{n}(0)$ and $\operatorname{Reg}_{1}^{n}(\lambda)$ are isomorphic to $k^{2 n}$. The path algebra $k Q_{1,1}$ acts on these isomorphic vector spaces by two actions that we denote respectively by $*$ and $\circ$. We consider the automorphism $\psi$ of the path algebra $k Q_{1,1}$ which sends $\varepsilon_{0}$ to $\varepsilon_{0}+\lambda \varepsilon_{1}$. For every $\sigma$ in $k Q_{1,1}$ and every $m$ in $\operatorname{Reg}_{1,1}^{n}(0)$, $\psi(\sigma) * m=\sigma \circ m$. Roughly speaking what the automorphism $\psi$ does is the following: the arrow $\varepsilon_{0}$ acts as $J_{n}(0)$ on $\operatorname{Reg}_{1,1}^{n}(0)$, while the arrow $\varepsilon_{1}$ acts as the identity. Then $\psi\left(\varepsilon_{0}\right)$ acts as $J_{n}(0)+\lambda I d=J_{n}(\lambda)$. With this action $\operatorname{Reg}_{1,1}^{n}(0)$ is isomorphic to $\operatorname{Reg}_{1,1}^{n}(\lambda)$ (as $k Q_{11}$-module). In particular the two representations have the 
same quiver Grassmannians. This proves that they are right-equivalent in the sense of [15].

This concludes the proof of Proposition 4.

\subsection{Type $\tilde{A}_{p, 1}$}

We prove Proposition 3 for every $p \geq 2$. The duality functor $D$ sends a representation of $Q_{p, 1}$ to a representation of the opposite quiver $Q_{p, 1}^{o p}$. The symmetries of such quiver induce an isomorphism $M_{p}^{n}(\underline{[1, t]}) \simeq D M_{p}^{n}(\overline{[1, p+1-t]})$ and, for every dimension vector $\mathbf{e}=\left(e_{1}, \ldots, e_{p+1}\right)$, we have:

$$
\chi_{\mathbf{e}}\left(M_{p}^{n}(\underline{[1, t]})\right)=\chi_{\left(d_{p+1}-e_{p+1}, \ldots, d_{1}-e_{1}\right)}\left(M_{p}^{n}(\overline{[1, p+1-t]})\right)
$$

where $\mathbf{d}=\left(d_{1}, \ldots, d_{p+1}\right)$ is the dimension vector of $M_{p}^{n}([1, t])$. Then (7) follows from (6).

We prove (6). By Lemma 2, the representation $M_{p}^{n}(\overline{[1, t]})$ satisfies the hypotheses of Theorem 1 . In order to compute $\chi_{\mathbf{e}}\left(M_{p}^{n}(\overline{[1, t]})\right)$ we hence have to count sets $\left\{T_{1}, \ldots, T_{p+1}\right\}$ of subsets $T_{1}, \ldots, T_{t} \subset[1, n+1], T_{t+1}, \ldots, T_{p+1} \subset[1, n]$ such that: $\left|T_{i}\right|=e_{i}$ and $\varphi_{1}\left(T_{t+1}\right) \subset T_{t}, \varphi_{2}\left(T_{p+1}\right) \subset T_{1}$ and $T_{k} \subset T_{k-1}(k \neq t+1, k \neq p+1)$ where $\varphi_{1}, \varphi_{2}:[1, n] \rightarrow[1, n+1]$ are defined by $\varphi_{1}(k):=k$ and $\varphi_{2}(k):=k+1$ for every $k=1, \ldots, n$.

For a choice of the quadruple $\left\{T_{1}, T_{t}, T_{t+1}, T_{p+1}\right\}$ (this set could collapse to a quadruple in which two elements coincide but it does not make any difference in the sequel and we still refer to it as a quadruple) there are $\chi_{\mathbf{e}}([1, t])$ choices for $\left\{T_{2}, \ldots, T_{t-1}\right\}$ and $\chi_{\mathbf{e}}([t+1, p+1])$ choices for $\left\{T_{t+2}, \ldots, T_{p}\right\}$ such that $\left\{T_{1}, \ldots, T_{p+1}\right\}$ is a desired tuple.

We hence prove that the number of quadruples $\left\{T_{1}, T_{t}, T_{t+1}, T_{p+1}\right\}$ equals:

$$
\left(\begin{array}{c}
e_{1}-1 \\
e_{p+1}
\end{array}\right)\left(\begin{array}{c}
n+1-e_{t} \\
e_{1}-e_{t}
\end{array}\right)\left(\begin{array}{c}
n+1-e_{t+1} \\
e_{t}-e_{t+1}
\end{array}\right)\left(\begin{array}{c}
n-e_{p+1} \\
e_{t+1}-e_{p+1}
\end{array}\right)
$$

from which (6) follows. We hence have to count the number of quadruples $\left\{T_{1}, T_{t}, T_{t+1}, T_{p+1}\right\}$ of subsets $T_{t} \subset T_{1} \subset[1, n+1], T_{p+1} \subset T_{t+1} \subset[1, n]$ such that $\left|T_{i}\right|=e_{i}, \varphi_{1}\left(T_{t+1}\right) \subset T_{t}$ and $\varphi_{2}\left(T_{p+1}\right) \subset T_{1}$.

We need the following lemma.

Lemma 5 Let $n$ and $e$ be positive integers such that $1 \leq e \leq n$. As before, we denote by $c(J)$ the number of connected components of an e-element subset $J$ of $[1, n]$. For every integer $c$, we have

(1) the number of e-element subsets $J$ of $[1, n]$ such that $c(J)=c$ and $J$ contains $n$, is $\left(\begin{array}{l}e-1 \\ c-1\end{array}\right)\left(\begin{array}{l}n-e \\ c-1\end{array}\right)$;

(2) the number of e-element subsets $J$ of $[1, n]$ such that $c(J)=c$ and $J$ does not contain $n$ is $\left(\begin{array}{c}e-1 \\ c-1\end{array}\right)\left(\begin{array}{c}n-e \\ c\end{array}\right)$;

(3) for every $0 \leq r \leq q \leq p,\left(\begin{array}{c}p \\ q\end{array}\right)\left(\begin{array}{l}q \\ r\end{array}\right)=\left(\begin{array}{c}p \\ r\end{array}\right)\left(\begin{array}{c}p-r \\ q-r\end{array}\right)$. 
Proof The proof of Lemma 5 follows from Lemma 3 by an easy induction.

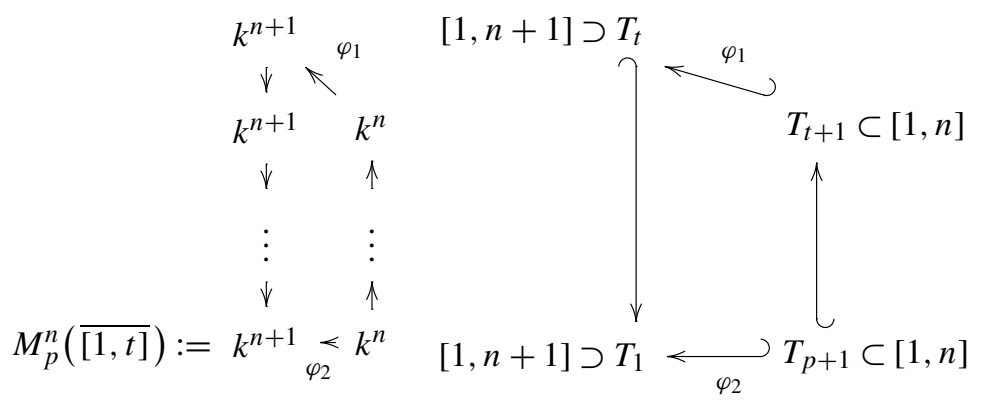

Let $T_{p+1}$ be an $e_{p+1}$-element subset of $[1, n]$ and let us count the number of desired quadruples $\left\{T_{1}, T_{t}, T_{t+1}, T_{p+1}\right\}$ containing $T_{p+1}$. We notice that $T_{1}$ contains both $\varphi_{1}\left(T_{p+1}\right)$ and $\varphi_{2}\left(T_{p+1}\right)$. In particular, if $c$ denotes the number of connected components of $T_{p+1}$, then $T_{1}$ must contain $c+e_{p+1}$ elements of $[1, n+1]$. We distinguish the two cases: either $T_{p+1}$ contains $n$ or it does not.

(1) If $T_{p+1}$ contains $n$ (by Lemma 5 there are $\left(\begin{array}{c}e_{p+1}-1 \\ c-1\end{array}\right)\left(\begin{array}{c}n-e_{p+1} \\ c-1\end{array}\right)$ choices for such subsets) then every possible $T_{1}$ contains the element $\varphi_{2}(n)=(n+1)$. Then there are $\left(\begin{array}{c}n+1-c-e_{p+1} \\ e_{1}-c-e_{p+1}\end{array}\right)$ choices for $T_{1}$. Now either $T_{t}$ contains $(n+1)$ or it does not. If it contains $(n+1)$ (there are $\left(\begin{array}{l}e_{1}-1-e_{p+1} \\ e_{t}-1-e_{p+1}\end{array}\right)$ choices for such sets) then there are $\left(\begin{array}{c}e_{t}-1-e_{p+1} \\ e_{t+1}-e_{p+1}\end{array}\right)$ choices for $T_{t+1}$; if $T_{t}$ does not contain $(n+1)$ (there are $\left(\begin{array}{c}e_{1}-1-e_{p+1} \\ e_{t}-e_{p+1}\end{array}\right)$ choices for such sets) then there are $\left(\begin{array}{c}e_{t}-e_{p+1} \\ t_{t+1}-e_{p+1}\end{array}\right)$ choices for $T_{t+1}$.

The number of quadruples $\left\{T_{1}, T_{t}, T_{t+1}, T_{p+1}\right\}$ such that $T_{p+1}$ contains $n$ is hence given by:

$$
\begin{aligned}
\sum_{c}\left(\begin{array}{c}
e_{p+1}-1 \\
c-1
\end{array}\right)\left(\begin{array}{c}
n-e_{p+1} \\
c-1
\end{array}\right)\left(\begin{array}{c}
n-e_{p+1}-(c-1) \\
e_{1}-e_{p+1}-c
\end{array}\right) \\
\times\left[\left(\begin{array}{c}
e_{1}-1-e_{p+1} \\
e_{t}-1-e_{p+1}
\end{array}\right)\left(\begin{array}{c}
e_{t}-1-e_{p+1} \\
e_{t+1}-e_{p+1}
\end{array}\right)+\left(\begin{array}{c}
e_{1}-1-e_{p+1} \\
e_{t}-e_{p+1}
\end{array}\right)\left(\begin{array}{c}
e_{t}-e_{p+1} \\
e_{t+1}-e_{p+1}
\end{array}\right)\right] \\
=\sum_{c}\left(\begin{array}{c}
e_{p+1}-1 \\
c-1
\end{array}\right)\left(\begin{array}{c}
n-e_{p+1} \\
c-1
\end{array}\right)\left(\begin{array}{c}
n-e_{p+1}-(c-1) \\
e_{1}-e_{p+1}-c
\end{array}\right) \\
\times\left[\left(\begin{array}{c}
e_{1}-1-e_{p+1} \\
e_{t+1}-e_{p+1}
\end{array}\right)\left(\begin{array}{c}
e_{1}-1-e_{t+1} \\
e_{t}-1-e_{t+1}
\end{array}\right)+\left(\begin{array}{c}
e_{1}-1-e_{p+1} \\
e_{t+1}-e_{p+1}
\end{array}\right)\left(\begin{array}{c}
e_{1}-1-e_{t+1} \\
e_{t}-e_{t+1}
\end{array}\right)\right] \\
=\sum_{c}\left(\begin{array}{c}
e_{p+1}-1 \\
c-1
\end{array}\right)\left(\begin{array}{c}
e_{1}-e_{p+1}-1 \\
c-1
\end{array}\right)\left(\begin{array}{c}
n-e_{p+1} \\
e_{1}-e_{p+1}-1
\end{array}\right) \\
\times\left(\begin{array}{c}
e_{1}-1-e_{p+1} \\
e_{t+1}-e_{p+1}
\end{array}\right)\left(\begin{array}{c}
e_{1}-e_{t+1} \\
e_{t}-e_{t+1}
\end{array}\right) \\
=\left(\begin{array}{c}
e_{1}-2 \\
e_{1}-e_{p+1}
\end{array}\right)\left(\begin{array}{c}
e_{1}-e_{t+1}-1 \\
e_{t}-e_{t+1}
\end{array}\right)\left(\begin{array}{c}
e_{1}-1-e_{p+1} \\
e_{t+1}-e_{p+1}
\end{array}\right) .
\end{aligned}
$$


In the first and third equality we have used part (3) of Lemma 5; in the last equality we have used the Vandermonde's identity.

(2) If $T_{p+1}$ does not contain $n$ (by Lemma 5 there are $\left(\begin{array}{c}e_{p+1}-1 \\ c-1\end{array}\right)\left(\begin{array}{c}n-e_{p+1} \\ c\end{array}\right)$ choices for such sets) then either $T_{1}$ contains $(n+1)$ or it does not. Since $T_{p+1}$ does not contain $n$, there are $\left(\begin{array}{c}n-c-e_{p+1} \\ e_{1}-1-c-e_{p+1}\end{array}\right)$ choices of sets $T_{1}$ containing $(n+1)$. In this case either $T_{t}$ contains $(n+1)$ (there are $\left(\begin{array}{c}e_{1}-1-e_{p+1} \\ e_{t}-1-e_{p+1}\end{array}\right)$ choices of such sets) or it does not (there are $\left(\begin{array}{c}e_{1}-1-e_{p+1} \\ e_{t}-e_{p+1}\end{array}\right)$ choices of such sets). If $T_{t}$ contains $(n+1)$ then there are $\left(\begin{array}{c}e_{t}-1-e_{p+1} \\ e_{t+1}-e_{p+1}\end{array}\right)$ choices for $T_{t+1}$. If $T_{t}$ does not contain $(n+1)$ then there are $\left(\begin{array}{c}e_{t}-e_{p+1} \\ e_{t+1}-e_{p+1}\end{array}\right)$ choices for $T_{t+1}$.

The number of quadruples $\left\{T_{1}, T_{t}, T_{t+1}, T_{p+1}\right\}$ such that $T_{p+1}$ does not contain $n$ and $T_{1}$ contains $(n+1)$ is hence given by:

$$
\begin{aligned}
\sum_{c}\left(\begin{array}{c}
e_{p+1}-1 \\
c-1
\end{array}\right)\left(\begin{array}{c}
n-e_{p+1} \\
c
\end{array}\right)\left(\begin{array}{c}
n-e_{p+1}-c \\
e_{1}-1-e_{p+1}-c
\end{array}\right) \\
\quad \times\left[\left(\begin{array}{c}
e_{1}-1-e_{p+1} \\
e_{t}-1-e_{p+1}
\end{array}\right)\left(\begin{array}{c}
e_{t}-1-e_{p+1} \\
e_{t+1}-e_{p+1}
\end{array}\right)+\left(\begin{array}{c}
e_{1}-1-e_{p+1} \\
e_{t}-e_{p+1}
\end{array}\right)\left(\begin{array}{c}
e_{t}-e_{p+1} \\
e_{t+1}-e_{p+1}
\end{array}\right)\right] \\
=\sum_{c}\left(\begin{array}{c}
e_{p+1}-1 \\
c-1
\end{array}\right)\left(\begin{array}{c}
e_{1}-e_{p+1}-1 \\
c
\end{array}\right)\left(\begin{array}{c}
n-e_{p+1} \\
e_{1}-e_{p+1}-1
\end{array}\right) \\
\quad \times\left(\begin{array}{c}
e_{1}-1-e_{p+1} \\
e_{t+1}-e_{p+1}
\end{array}\right)\left(\begin{array}{c}
e_{1}-e_{t+1} \\
e_{t}-e_{t+1}
\end{array}\right) \\
=\left(\begin{array}{c}
e_{1}-2 \\
e_{1}-e_{p+1}-2
\end{array}\right)\left(\begin{array}{c}
n-e_{p+1} \\
e_{1}-e_{p+1}-1
\end{array}\right)\left(\begin{array}{c}
e_{1}-1-e_{p+1} \\
e_{t+1}-e_{p+1}
\end{array}\right)\left(\begin{array}{c}
e_{1}-e_{t+1} \\
e_{t}-e_{t+1}
\end{array}\right) .
\end{aligned}
$$

By summing up (14) and (15) and by applying Lemma 3 we get

$$
\left(\begin{array}{c}
e_{1}-1 \\
e_{p+1}
\end{array}\right)\left(\begin{array}{c}
n-e_{t+1} \\
e_{1}-e_{t+1}-1
\end{array}\right)\left(\begin{array}{c}
n-e_{p+1} \\
e_{t+1}-e_{p+1}
\end{array}\right)\left(\begin{array}{c}
e_{1}-e_{t+1} \\
e_{t}-e_{t+1}
\end{array}\right) .
$$

If $T_{1}$ does not contain $(n+1)$ (there are $\left(\begin{array}{c}n-c-e_{p+1} \\ e_{1}-c-e_{p+1}\end{array}\right)$ choices of such sets) then there are $\left(\begin{array}{c}e_{1}-e_{p+1} \\ e_{t}-e_{p+1}\end{array}\right)$ choices for $T_{t}$ and $\left(\begin{array}{c}e_{t}-e_{p+1} \\ e_{t+1}-e_{p+1}\end{array}\right)$ choices for $T_{t+1}$. The number of quadruples $\left\{T_{1}, T_{t}, T_{t+1}, T_{p+1}\right\}$ such that $T_{p+1}$ does not contain $n$ and $T_{1}$ does not contain $(n+1)$ is hence given by:

$$
\begin{aligned}
& \sum_{c}\left(\begin{array}{c}
e_{p+1}-1 \\
c-1
\end{array}\right)\left(\begin{array}{c}
n-e_{p+1} \\
c
\end{array}\right)\left(\begin{array}{c}
n-e_{p+1}-c \\
e_{1}-e_{p+1}-c
\end{array}\right)\left(\begin{array}{c}
e_{1}-e_{p+1} \\
e_{t}-e_{p+1}
\end{array}\right)\left(\begin{array}{c}
e_{t}-e_{p+1} \\
e_{t+1}-e_{p+1}
\end{array}\right) \\
& =\sum_{c}\left(\begin{array}{c}
e_{p+1}-1 \\
c-1
\end{array}\right)\left(\begin{array}{c}
e_{1}-e_{p+1} \\
c
\end{array}\right)\left(\begin{array}{c}
n-e_{p+1} \\
e_{1}-e_{p+1}
\end{array}\right)\left(\begin{array}{c}
e_{1}-e_{t+1} \\
e_{t}-e_{t+1}
\end{array}\right)\left(\begin{array}{c}
e_{1}-e_{p+1} \\
e_{t+1}-e_{p+1}
\end{array}\right) \\
& =\left(\begin{array}{c}
e_{1}-1 \\
e_{p+1}
\end{array}\right)\left(\begin{array}{c}
n-e_{p+1} \\
e_{1}-e_{p+1}
\end{array}\right)\left(\begin{array}{c}
e_{1}-e_{t+1} \\
e_{t}-e_{t+1}
\end{array}\right)\left(\begin{array}{c}
e_{1}-e_{p+1} \\
e_{t+1}-e_{p+1}
\end{array}\right)
\end{aligned}
$$




$$
=\left(\begin{array}{c}
e_{1}-1 \\
e_{p+1}
\end{array}\right)\left(\begin{array}{c}
n-e_{t+1} \\
e_{1}-e_{t+1}
\end{array}\right)\left(\begin{array}{c}
n-e_{p+1} \\
e_{t+1}-e_{p+1}
\end{array}\right)\left(\begin{array}{c}
e_{1}-e_{t+1} \\
e_{t}-e_{t+1}
\end{array}\right) .
$$

By summing up (16) and (17) and by applying Lemma 3 we get the desired (13).

We now prove (8). As for the case $p=1$ (see Lemma 4), the variety $\operatorname{Gr}_{\mathbf{e}}\left(\operatorname{Reg}_{p}^{n}(\lambda)\right.$ ) equals the variety $\operatorname{Gr}_{\mathbf{e}}\left(\operatorname{Reg}_{p}^{n}(0)\right)$ for every $\lambda \in k$. Indeed let us denote by $\circ$ and by * respectively the action of $A=k Q_{p, 1}$ on $\operatorname{Reg}_{p}^{n}(\lambda)$ and on $\operatorname{Reg}_{p}^{n}(0)$. We consider the automorphism $\psi$ of the path algebra $k Q_{p, 1}$ which sends $\varepsilon_{0}$ to $\lambda \pi+\varepsilon_{0}$ where $\pi:=\varepsilon_{1} \circ \cdots \circ \varepsilon_{p}$ is the longest path of $Q_{p, 1}$. As vector spaces, $\operatorname{Reg}_{p}^{n}(0)$ and $\operatorname{Reg}_{p}^{n}(\lambda)$ are isomorphic. Then for every $\pi$ in $A$ and every $m$ in $\operatorname{Reg}_{p}^{n}(0), \psi(\pi) * m=\pi \circ m$. This proves that they are right-equivalent in the sense of [15].

$$
\begin{aligned}
& k^{n} \stackrel{=}{\leftarrow} \stackrel{=}{\leftarrow} k^{n} \\
& =\downarrow \quad \uparrow= \\
& \operatorname{Reg}_{p}^{n}(\lambda):=k^{n} \leftarrow{ }_{J_{n}(\lambda)} k^{n}
\end{aligned}
$$

We thus assume that $\lambda=0$. In this case the representation $\operatorname{Reg}_{p}^{n}(0)$ is an orientable string module by Lemma 2 and we can therefore apply Theorem 1 . The Euler-Poincaré characteristic of $\operatorname{Gr}_{\mathbf{e}}\left(\operatorname{Reg}_{p}^{n}(0)\right)$ is hence the number of $(p+1)$-tuples $\left\{T_{1}, \ldots, T_{p+1}\right\}$ of subsets $T_{i} \subset[1, n]$ of cardinality $\left|T_{i}\right|=e_{i}$ such that $T_{i+1} \subset T_{i}$ for $i=1, \ldots, p$ and $J_{n}(0)\left(T_{p+1}\right) \subset T_{1}$ where $J_{n}(0):[1, n] \rightarrow[1, n] \cup\{0\}$ is the map which sends $k$ to $k-1, k \in[1, n]$. The choice of the couple $\left\{T_{1}, T_{p+1}\right\}$ determines the choice of $\left(\begin{array}{l}e_{1}-e_{p+1} \\ e_{2}-e_{p+1}\end{array}\right)$ choices for $T_{2}$. For every such choice there are $\left(\begin{array}{l}e_{2}-e_{p+1} \\ e_{3}-e_{p+1}\end{array}\right)$ choices for $T_{3}$, and so on. For every choice of $\left\{T_{1}, T_{p+1}\right\}$ there are hence $\chi_{\mathbf{e}}([1, p+1])=\prod_{k=1}^{p-1}\left(\begin{array}{c}e_{k}-e_{p+1} \\ e_{k+1}-e_{p+1}\end{array}\right)$ choices for $\left\{T_{2}, \ldots, T_{p}\right\}$. The number of couples $\left\{T_{1}, T_{p+1}\right\}$ equals $\chi_{\left(e_{1}, e_{p+1}\right)}\left(\operatorname{Reg}_{1}^{n}(0)\right)$. It remains to prove that:

$$
\chi_{\left(e_{1}, e_{2}\right)}\left(\operatorname{Reg}_{1}^{n}(0)\right)=\left(\begin{array}{l}
e_{1} \\
e_{2}
\end{array}\right)\left(\begin{array}{c}
n-e_{2} \\
e_{1}-e_{2}
\end{array}\right)
$$

which has already been noticed in Proposition 4.

Acknowledgements I thank Professor B. Keller for his kind hospitality and for useful discussions on this topic during my stay in Paris. I thank Professor J. Schröer for many conversations about orientable string modules and for his support during my stay in Bonn. I thank Professor A. Zelevinsky for his advice on the structure of this paper. This paper was accepted when I had a post-doc position at "Sapienza Universitá di Roma” (Rome, Italy). I thank Professor C. De Concini for his support.

\section{References}

1. Assem, I., Reutenauer, C., Smith, D.: Frises. ArXiv e-prints, June (2009)

2. Assem, I., Simson, D., Skowroński, A.: Elements of the Representation Theory of Associative Algebras, Vol. 1. London Mathematical Society Student Texts, vol. 65. Cambridge University Press, Cambridge (2006). Techniques of representation theory 
3. Auslander, M., Reiten, I., Smalø, S.O.: Representation Theory of Artin Algebras. Cambridge Studies in Advanced Mathematics, vol. 36. Cambridge University Press, Cambridge (1997). Corrected reprint of the 1995 original

4. Białynicki-Birula, A.: Some theorems on actions of algebraic groups. Ann. Math. (2) 98, 480-497 (1973)

5. Caldero, Ph., Chapoton, F.: Cluster algebras as Hall algebras of quiver representations. Comment. Math. Helv. 81(3), 595-616 (2006)

6. Caldero, Ph., Keller, B.: From triangulated categories to cluster algebras. II. Ann. Sci. École Norm. Sup. (4) 39(6), 983-1009 (2006)

7. Caldero, Ph., Keller, B.: From triangulated categories to cluster algebras. Invent. Math. 172(1), 169$211(2008)$

8. Caldero, Ph., Reineke, M.: On the quiver Grassmannian in the acyclic case. J. Pure Appl. Algebra 212(11), 2369-2380 (2008)

9. Caldero, Ph., Zelevinsky, A.: Laurent expansions in cluster algebras via quiver representations. Mosc. Math. J. 6(3), 411-429 (2006)

10. Cerulli Irelli, G.: Structural theory of rank three cluster algebras of affine type. $\mathrm{PhD}$ thesis, Università degli studi di Padova (2008). Also available as http://paduaresearch.cab.unipd.it/734/

11. Cerulli Irelli, G.: Canonically positive basis of cluster algebras of type $A_{2}^{(1)}$ (2009). arXiv:0904.2543

12. Cerulli Irelli, G., Esposito, F.: Geometry of quiver Grassmannians of Kronecker type and canonical basis of cluster algebras (March 2010). arXiv:1003.3037

13. Chriss, N., Ginzburg, V.: Representation Theory and Complex Geometry. Birkhäuser, Boston (1997)

14. Crawley-Boevey, W.W.: Maps between representations of zero-relation algebras. J. Algebra 126(2), 259-263 (1989)

15. Derksen, H., Weyman, J., Zelevinsky, A.: Quivers with potentials and their representations. I. Mutations. Sel. Math. (N.S.) 14(1), 59-119 (2008)

16. Derksen, H., Weyman, J., Zelevinsky, A.: Quivers with potentials and their representations II: Applications to cluster algebras (2009)

17. Dupont, G.: Generic variables in acyclic cluster algebras (2008). arXiv:0811.2909

18. Fomin, S., Zelevinsky, A.: Cluster algebras. I. Foundations. J. Am. Math. Soc. 15(2), 497-529 (2002) (electronic)

19. Fomin, S., Zelevinsky A.: Cluster algebras. II. Finite type classification. Invent. Math. 154(1), 63-121 (2003)

20. Fomin, S., Zelevinsky A.: Cluster algebras. IV. Coefficients. Compos. Math. 143(1), 112-164 (2007)

21. Gabriel, P.: The universal cover of a representation-finite algebra. In: Representations of Algebras, Puebla, 1980. Lecture Notes in Math., vol. 903, pp. 68-105. Springer, Berlin (1981)

22. Musiker, G., Schiffler, R., Williams, L.: Positivity for cluster algebras from surfaces (2009)

23. H. Nakajima: Quiver varieties and cluster algebras. ArXiv e-prints, April (2009)

24. Ringel, C.M.: Exceptional modules are tree modules. In: Proceedings of the Sixth Conference of the International Linear Algebra Society, Chemnitz, 1996, vols. 275/276, pp. 471-493 (1998) 